\section{The illusion of certainty}

\section{Luca Iaboli, ${ }^{1}$ Luana Caselli² \\ ${ }^{1}$ Accident and Emergency Department, Local Health Unit of Modena; ${ }^{2}$ Private Graphic Designer, Montale di Castelnuovo Rangone (MO), Italy}

In this world nothing can be said to be certain, except death and taxes. Benjamin Franklin

In everyday life we have to make choices, which involve a degree of uncertainty and risk, however many doctors are not able to take proper decisions and fail to understand the advantages and the limitations of the available medical evidence. One of the main reasons for this deficiency is statistical illiteracy, that is, the inability to understand the risk and the probabilities that lie behind the decisions mainly due to poor reporting of information.

For example, saying that a statin reduces heart attack by $36 \%$ seems to be very encouraging. However, $36 \%$ actually means that the risk of heart attack in those who take the drug is only $2 \%$ compared to $3 \%$ in those who do not take it! ${ }^{1}$ Therefore, using relative risk reduction is good for increasing sales but inaccurate to understand the actual efficacy of a drug. Moreover, nowadays, to answer questions of clinical practice, doctors need more than ever to be able to access original research reports and scientific publications and cannot base their knowledge solely on abstracts, summaries of meta-analyses and guidelines usually pertaining to a limited number of health conditions. This requires the readers to understand a wealth of basic statistical concepts, such as relative risk, absolute risk, number needed to treat, number needed to harm and odds ratio that enable to critically evaluate the design, the implementation and the analysis of each study and subsequently to interpret the results correctly.

Studies have shown that doctors are able to understand the analysis and interpretation of the results of only $21 \%$ of scientific articles. ${ }^{2}$ Statistical literacy of most doctors is so poor that you cannot expect that they draw the right conclusions from statistical analysis of studies published in medical journals and it does not seem that understanding has improved compared to forty years ago. ${ }^{3,4}$

A statistical culture provides physicians the skills to understand numbers and it applies to every decision, from prescribing a drug to treat hypertension or refer a patient to a CT in case of pulmonary thromboembolism suspicion.

Imagine a blood donor, without risk behavior, worried about a positive HIV selftest result, asking you the following question: What is the probability I'm HIV-infected? $100 \%$, 99\%, 50\%, or maybe less? You are up-to-date and you know that the HIV test performed by the patient has a sensitivity of $100 \%$ and a specificity of $99.8 \%$. Moreover, HIV prevalence in his population is $0.01 \%$. The answer is the positive predictive power of the HIV test, a basic statistical concept that every professional should handle.

In contrast, although they know exactly the weak points of a clinical trial and the prevalence of a given disease, doctors are not able to infer from this information the probability that a patient found positive actually has the disease. ${ }^{5}$

This issue is particularly relevant as since 1st December, the HIV self-test is on sale in the italian pharmacies.

This test, proposed by the WHO, is certainly good in places where the prevalence of the disease is high (e.g., in some groups in Africa, $15 \%$ of adults are infected): there, the test can actually make a difference. But in high-income countries the prevalence of the disease is much lower and the problem about HIV self-testing is that also people at low risk of infection might think to do the test.

The prevalence of HIV infection in a population without risk factors is about 1 out of 10000 people $(0.01 \%$, as mentioned above). This means that 9999 people are not
Correspondence: Luca Iaboli, Accident and Emergency Department, Local Health Unit of Modena, via S. Giovanni del cantone 23, 41121 Modena, Italy.

Tel: +39.348.2635473

E-mail: liaboli@hotmail.com

Key words: Statistics; Scientific graphic novel; Risks; Informed choices; Decision making.

Contributions: all authors contributed equally to this work.

Conflict of interest: Iaboli and Caselli are authors of Doctor G, a scientific graphic novel to help health professionals to understand basic health statistics.

This work is licensed under a Creative Commons Attribution 4.0 License (by-nc 4.0).

(C) Copyright L. Iaboli and L.Caselli, 2017

Licensee PAGEPress, Italy

Emergency Care Journal 2017; 13:6499

doi:10.4081/ecj.2017.6499

infected and one is HIV-positive. The latter will likely be test-positive (the test has a 100\% sensitivity). Of the 9999 healthy people, the test will correctly identify 9979 as non-infected (the test specificity is $99.8 \%$ ), while 20 people will be false positives, i.e., they will test positive even though they are not infected $(9999-9979=20)$. In practice, 21 people will get a positive test result, but only 1 will have HIV. Therefore, you should answer your patient that the probability he's

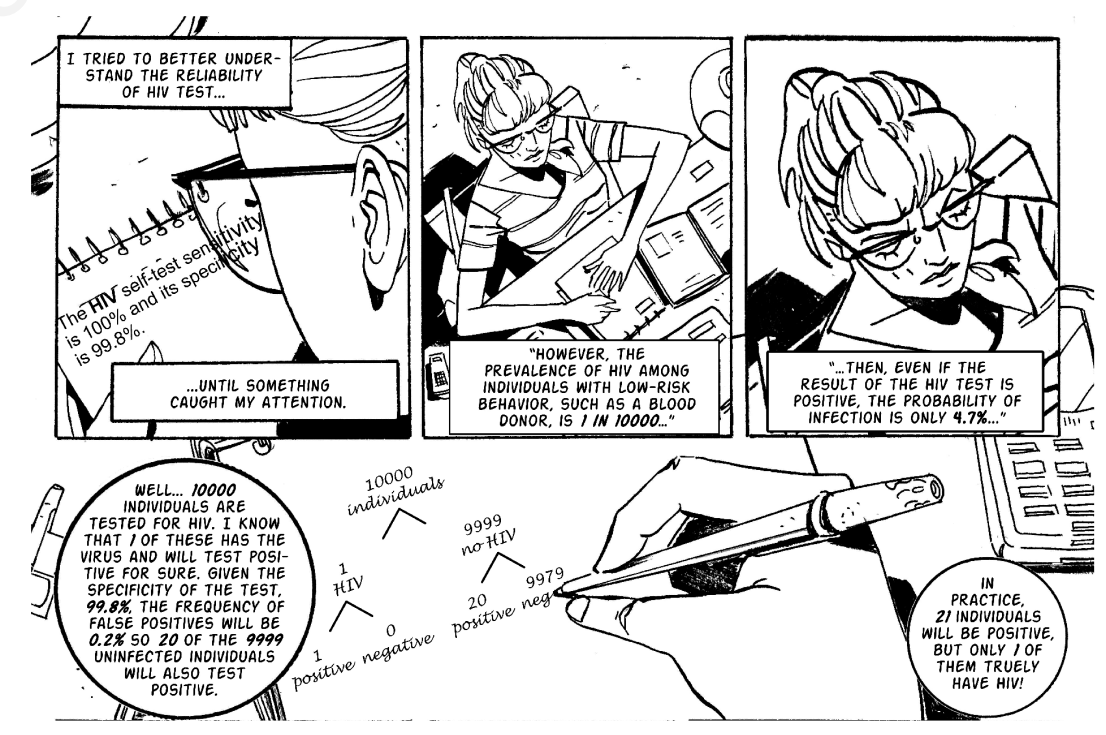

Figure 1. How to calculate the positive predictive power of HIV self-test given an HIV infection prevalence of $0.01 \%$, test sensitivity $100 \%$ and test specificity $99.8 \%$. 
HIV-infected given his positive test result is 1 out of 21 , that is, $4.7 \%$ (Figure 1 ).

The heart of this issue is the illusion of certainty: we are used to trust tests too much ignoring that even the best test is not perfect and the results should be interpreted carefully.

Statistical thinking is not for few chosen people, but rather a tool for everyone. To ensure that it is within everyone's reach, statistical information has to be presented in a clear and intuitive way. Doctors don't have to be afraid of statistics but be able to use it to make proper decisions. Basic sta- tistics is the key to better cope with numbers in everyday life and effective risk communication can improve the quality of healthcare and contribute to the cultural and professional growth of the world of emergency.

\section{References}

1. Gigerenzer G, Gaissmaier W, KurzMilcke E, et al. Helping doctors and patients make sense of health statistics. Psychol Sci Public Interest 2007;8:5396.
2. Windish DM, Huot SJ, Green ML. Medicine residents' understanding of the biostatistics and results in the medical literature. JAMA 2007;298:101022.

3. Wulff HR, Andersen B, Brandenhoff P, Guttler F. What do doctors know about statistics? Stat Med 1987;6:3-10.

4. Martyn C. Risky business: doctors' understanding of statistics. BMJ 2014;349:g5619.

5. Gigerenzer G, Edwards A. Simple tools for understanding risks: from innumeracy to insight. BMJ 2003;327:741-4. 